\title{
Prediction of Relative Permeability and Capillary Pressure from Pore-Scale Modelling
}

\author{
$\emptyset$ REN, Pål-Eric, Statoil Research Centre \\ BAKKE, Stig, Statoil Resarch Centre \\ SCANDELLARI NILSEN, Laura, Stavanger University \\ HENRIQUEZ, Adolfo, Statoil a.s.
}

Paper presented at the 5th European Conference on Mathematics of Oil Recovery, Leoben, Austria, 3-6 Sept. 1996

\begin{abstract}
The present paper presents a new method for constructing realistic and fully characterised 3-D network representations of the pore space for actual sandstones. The essence of our approach is to build sandstone models which are analogs of real sandstones by simulating the results of the main sandstone forming geological processes sedimentation, compaction, and diagenesis. Singleand two-phase flow (drainage) in the generated pore networks are simulated using a network model which accounts for both viscous and capillary forces. Predicted absolute and relative permeabilities are compared with measured values and found to be in good agreement.
\end{abstract}

\section{INTRODUCTION}

Macroscopic multiphase flow in porous media is usually described in terms of Darcy's law and measured or empirically derived saturation dependent relationships for phase relative permeability and capillary pressure. These relationships are often tedious and difficult to determine experimentally. Furthermore, the rock samples which are available to perform measurements on constitute only a tiny fraction of the total reservoir volume. A degree of uncertainty is therefore always associated with relative permeability and capillary pressure input data. This prevents reliable predictions of production profiles and limits the ability of reservoir simulators to accurately predict oil recovery.

Relative permeability and capillary pressure are averaged transport properties which represent the physical processes occurring on the microscopic or pore-scale. In principle, it should therefore be possible to determine these properties by appropriately averaging the equations describing the pore level processes. This approach, known as network modelling, requires a detailed understanding of the pore level physics and a complete description of the morphology of the pore space. Since the pioneering work of Fatt ${ }^{1 \cdot 3}$, network models have been used extensively to study both two- and three-phase flow in simple or idealised porous media-25. The extension of network modelling techniques to real porous media is complicated by the difficulty of adequately describing the complex microstructure of the pore space for actual porous media.

Traditionally, information about the microstructure of reservoir rocks are obtained from mercury porosimetry and image analysis of thin section images. Mercury injection data provide information about pore entry sizes whilst 2-D thin sections provide statistical data on scalar properties such as porosity, grain sizes, and mineralogy. They do not, however, provide direct information on 3-D vector quantities such as the architecture of the pore space and the geometry of pore bodies and throats. Traditional pore structure analyses are thus insufficient to allow construction of 3-D pore networks which accurately represent the microstructure of the rock.

Advanced techniques such as micro- $\mathrm{CT}^{26}$ and serial sectioning combined with BSE imaging of the sections ${ }^{27.28}$ do provide a detailed description of the 3-D microstructure of rocks. These techniques are, however, expensive and not readily available. Bryant et al. ${ }^{29}$ constructed 3-D pore networks by simulating the results of compaction and cementation in a packing of equal spheres constructed by Finney ${ }^{30}$. The method of reconstructed porous media ${ }^{31}$ can also be used to construct 3-D replica of the pore space.

In the present work, we generate realistic 3-D network representations of the pore space for actual sandstones by simulating the results of the main sandstone forming processes sedimentation, compaction, and diagenesis. We then describe a two-phase network model which accounts for both viscous and capillary forces. The model is used to predict permeability and drainage relative permeability for the generated pore networks. Comparisons with measured values show that the model provides a correct macroscopic description of both single- and two-phase flow. 


\section{MODELLING OF SANDSTONES}

Sandstones are the end products of a series of complex geological processes which, schematically described, starts with erosion of quartz-bearing rocks, followed by sand grain transport via air, water or ice, and, finally, deposition of grains in sedimentary basins. The deposited grains may be reworked by one or several cycles of erosion, transport, and sedimentation. The sandstone generation process is completed with compaction and different diagenetic processes. We do not attempt to model the detailed dynamics of the complex sandstone forming processes. Instead, we simulate the results of these processes in three main steps; (i) sand grain sedimentation, (ii) compaction, and (iii) diagenesis.

Input Data. The required input data for the modelling are readily obtained from image analysis of thin section images (Fig. 1) and include porosity, grain-size distribution, amount of cement, and clay mineralogy. In the present work, these data were obtained from petrographical thin section images based on a combination of BSE image, cathodoluminiscence image and $\mathrm{X}$-ray images of characteristic elements ${ }^{32}$. Such images provide information about detrital grain-size distribution, quartz cement thickness, carbonate cement, feldspar contents, and clay mineralogy.

For simplicity, all the detrital sand grains are assumed to be spherical quartz grains whilst all the cement is treated as concentric quartz overgrowth on the quartz grains. We do not distinguish between different types of clay minerals but treat all the clay as a mixture of mainly kaolinite and some illite.

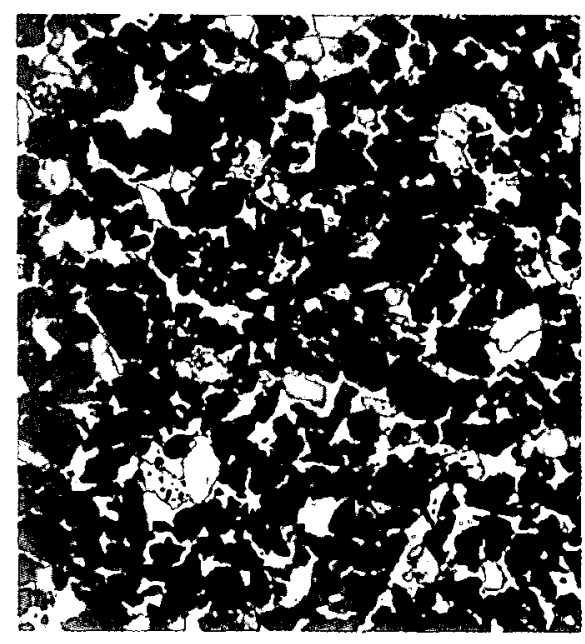

Fig. 1. BSE image of a thin section from a reservoir sandstone. The pore space is white, feldspar is light grey, quartz is medium grey, and clay minerals are dark grey.

Sedimentation. Modelling of the sedimentation process commences with the generation of a grain-size distribution curve. All the grain sizes recorded from the thin section analysis are numbered and ordered according to size, as shown in Fig. 2. To mimic the stochastic nature of the geological processes which affect the deposition of individual sand grains, grains are randomly chosen from the grain-size distribution curve and deposited in a predefined sandbed basin (bounding box).

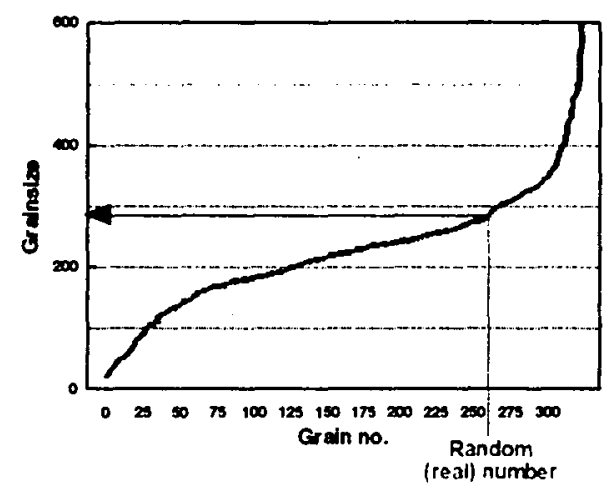

Fig.2. Example illustrating how grains are randomly selected from a numbered and ordered list of measured grain sizes.

The exact location in the sandbed where each new sand grain is deposited depends on whether the sedimentation process occurs in a low or high energy environment. For low energy sedimentation, it is assumed that the horizontal velocity of the new sand grain is zero and that no lateral forces affect the sandbed. In this case, each new randomly selected sand grain is dropped onto the sandbed from a random $X, Y$ position. Upon hitting the sandbed, it rolls down the maximum local gradient until it hits a stable position in a local minimum (Fig. 3). This leads to an undulating sandbed surface with local peaks and valleys.

Sedimentation of sand grains in extreme low energy basins is, however, rare. Sandstones are usually the results of high energy depositional events (low energy deposition results in claystones and siltstones). Sand grains precipitate from water or air suspension when the lateral velocity decreases below a threshold value which is proportional to the grain size $\mathrm{s}^{33}$. The sandbed is usually influenced by lateral forces like streams and waves, and gravity in the case of dipping beds. These lateral forces move individual sand grains around until they settle in a stable position where the effect of the transporting forces is at a minimum.

High energy sedimentation is modelled by placing each new randomly selected sand grain at the lowest available position (global minimum) in the sandbed (Fig. 4). In contrast to low energy sedimentation, high energy sedimentation leads to an almost flat sandbed surface. The porosity values for the sandbeds depicted in Figs. 3 and 4 show that high energy (global minimum) sedimentation results in a denser sandbed than low energy (local minimum) sedimentation. In general, modelling of fine sand or silt deposition is carried out as low energy sedimentation whereas sand and coarse sand deposition is modelled as high energy sedimentation. Further details of the modelling are given elsewhere ${ }^{34}$. 


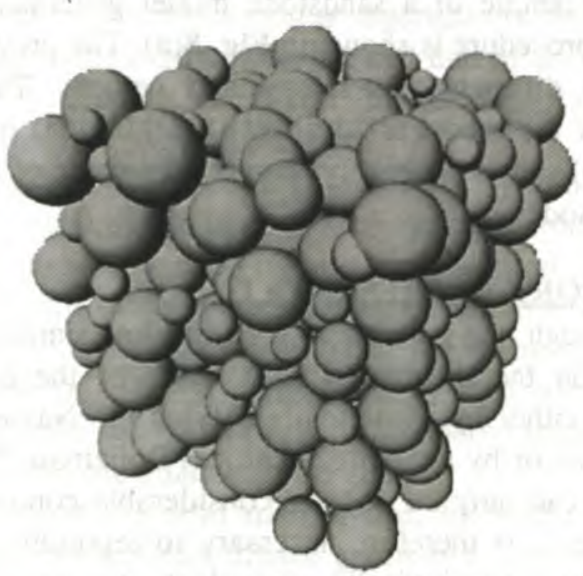

Fig. 3. Low energy basin (local minimum) sedimentation results in an open sandbed with porosity $\phi=37.8 \%$.

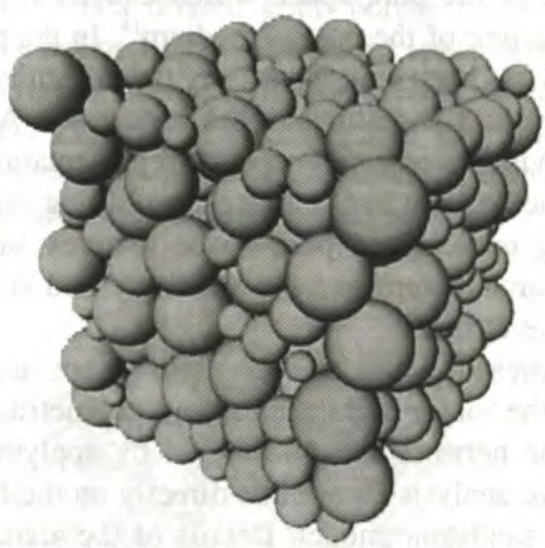

Fig. 4. High energy basin (global minimum) sedimentation, using the same inputs as in Fig. 3, results in a denser sandbed with porosity $\phi=35.3 \%$.

Compaction. Compaction, or bulk volume reduction, in response to vertical stress from the overburden is an important cause of porosity reduction in sandstones undergoing burial. At shallow depths, compaction is by mechanical processes such as grain rearrangements and fracturing. At greater depths, grain interpenetration as a result of pressure dissolution in the presence of $\mathrm{K}_{2} \mathrm{O}$-bearing minerals ${ }^{35}$ is the dominant compaction mechanism. At present, we consider only the pressure dissolution compaction mechanism.

The results of this compaction mechanism are modelled in a manner similar to that described by Bryant $e t$ $a l .{ }^{29}$. The $\mathrm{Z}$ co-ordinate of every sand grain centres are shifted vertically downwards by an amount which is proportional to the original Z-position, i.e.

$$
Z=Z_{o}(1-\lambda)
$$

where $Z_{o}$ is the original value of the $Z$ co-ordinate, $Z$ is the value of the co-ordinate after compaction, and $\lambda$ is a compaction factor which measures the degree of compaction $(1<\lambda<0)$. Fig. 5 shows the results of compaction simulation for different compaction factors. The reduction in porosity is clearly observed. Simulation of compaction causes grains to interpenetrate. This is a direct analog of pressure dissolution at grain contacts ${ }^{29}$. For simplicity, we assume that the interpenetration results in no deformation of the grains. The material corresponding to the grain interpenetration may be dissolved and washed out of the system or it may be re-precipitated as quartz cement overgrowth (see below).

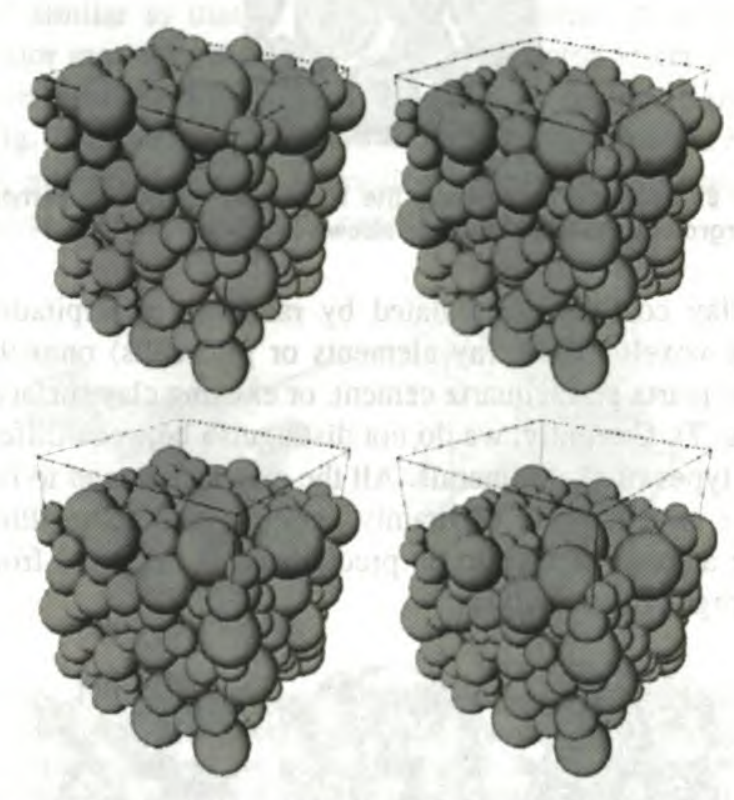

Fig. 5. Effect of linear compaction modelling for compaction factors equal to $0.0(\phi=31.2 \%), 0.1(\phi=26.1 \%), 0,2(\phi=20.8 \%)$ and $0.3(\phi=15.7 \%)$.

Diagenesis. Diagenetic processes sometimes introduce heterogeneities which totally overprint the heterogeneity pattern of the primary structures of the sandstone. Diagenetic processes may be very complex, involving several phases of dissolution of minerals and cement overgrowth, often in interaction with compaction, as a result of changing geological conditions. Currently, only a simplified subset of known diagenetic processes are modelled: quartz cement overgrowth and subsequent clay coating of the free surface.

In thin sections, quartz cement is often observed to be precipitated on the quartz sand grains as approximately concentric overgrowths. This is simulated by increasing the radius of all the sand grains uniformly ${ }^{29}$

$$
R_{i}=R_{i, o}+\Delta R
$$

where $R_{i, o}$ is the original radius of the $i$ th grain, $R_{i}$ is the new radius after grain growth, and $\Delta R$ is the cement thickness. Currently, we assume that the same value of $\Delta R$ applies to all the sand grains. However, the method is readily extended to the case in which the radii of different grains increase with different amounts. A schematic of the grain overgrowth simulation is depicted in Fig. 6.

Depending on the prevailing physical conditions, the amount of quartz cement overgrowth may be equal to the 
volume loss from the grain interpenetration during compaction or it may be specified by a cement thickness input parameter. The latter mimics the migration of $\mathrm{SiO}_{2}$ to or from the sandstone model.

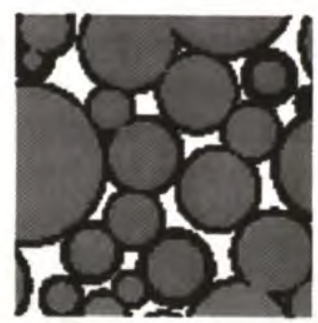

Fig. 6. Example illustrating the modelling of quartz cement overgrowth. Quartz cement is shown in black.

Clay coating is simulated by randomly precipitating clay voxels (3-D array elements or grid cells) onto the free quartz grain, quartz cement, or existing clay surfaces (Fig. 7). Currently, we do not distinguish between different types of clay minerals. All the clay is assumed to behave as a mixture of mainly kaolinite and some illite. The amount of clay to be precipitated is extracted from petrographical analysis.

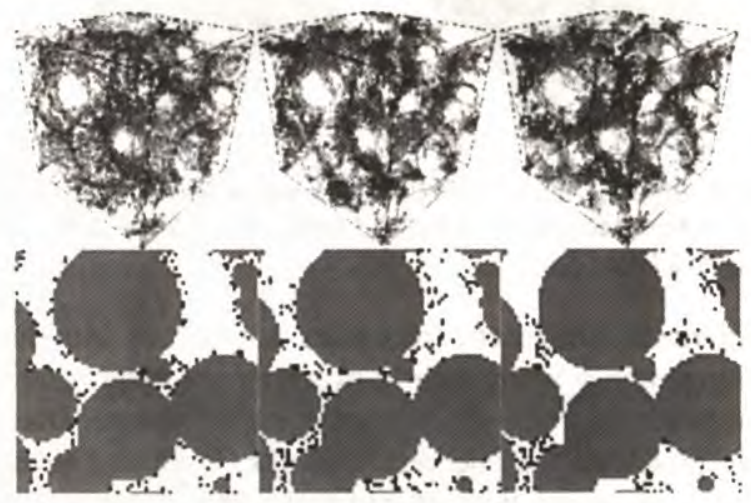

Fig. 7. Schematic illustration of the modelling of clay coating. Increasing clay clustering towards the right.

In thin sections, clay minerals are sometimes observed to be concentrated in a few pores whilst the rest of the pores are almost clay free. This type of clay coating is simulated using a clustering routine which increases the likelihood of new clay voxels to be precipitated on already existing clay voxels, as illustrated in Fig. 7.
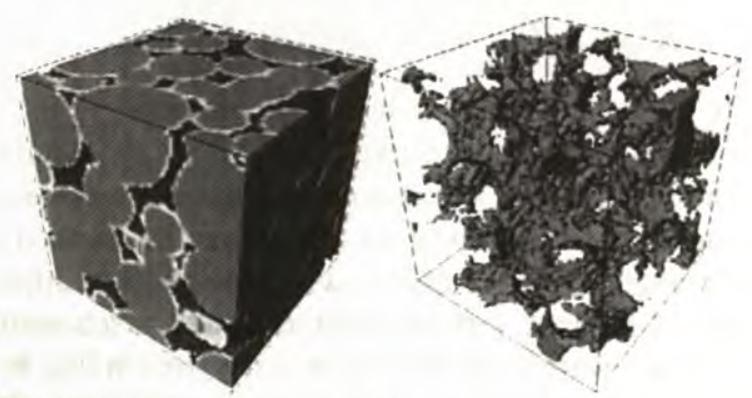

Fig. 8. Isosurface representation of (a) a sandstone model and (b) its pore space generated from the sandbed shown in Fig. 4.
An example of a sandstone model generated by the above procedure is shown in Fig. 8(a). The grey shading reflects different mineralogy and cement. The linear scale of the model is 70 voxels (or $0.07 \mathrm{~cm}$ ) on a side. Fig 8(b) shows the highly chaotic pore space of the sandstone model.

\section{NETWORK REPRESENTATION}

Although it is possible to perform flow simulations directly on the disordered pore space of the sandstone model, either by numerically solving the Navier-Stokes equations or by applying a Lattice- Boltzmann ${ }^{36}$ simulation, it can only be done at considerable computational expense. It is therefore necessary to represent the pore space by a topologically equivalent network which retains all the essential features relevant to fluid flow.

In principle, it is possible to construct a network representation of the pore space which exactly replicate the microstructure of the porous medium ${ }^{16}$. In the past, however, this has been complicated by the difficulty of measuring various $3-D$ features of the pore space. As a result, most previously reported network representations of real porous media have had to make simplifying assumptions regarding unmeasured pore space features such as the network architecture and the geometry and size of pore bodies and throats.

In the present work, no such assumptions are invoked. Instead, the major architectural and geometrical properties of the network are determined by applying various 3-D image analysis algorithms directly on the fully characterised sandstone model. Details of the algorithms are given elsewhere ${ }^{34}$.

Network architecture, the skeleton. The pore space architecture is defined as a complete 3-D characterisation of the network structure of the pore space. Serra ${ }^{37}$ and Adler $^{31}$ define the skeleton of the pore space as the set of points (voxels) at equal distances from two or more points of the solid wall. The skeleton may thus be thought of as a spatial representation of the deformation retract, or the centre line, of the pore network. This spatial centre line contains points where 2 or more lines meet. These meeting points are the network nodes (approximate centre of pore bodies) which are connected to other nodes via links or pore throats.

In 2-D imag̀e analysis, skeleton extraction from binary images is carried out by means of thinning algorithms ${ }^{38}$. 2-D thinning algorithms are mainly based on deleting or preserving the analysed pixel according to the rules for the binary 1- and 0 -pattern of its surrounding 8 neighbour pixels. In $2-D$, the number of possible 0 - and 1-combinations around one pixel is $2^{8}=256$. In 3-D, the number of combinations of 0 's and 1's for the 26 neighbours surrounding one voxel is $2^{26}=67108864$, which makes definition of a complete set of rules for a 3-D thinning algorithm very tedious.

Thovert et al. ${ }^{39}$ succeeded in developing a 3-D thinning algorithm which worked well on their models. Attempts 
were made to develop a similar algorithm in this work. However, visual examination showed that in some complex voxel junctions, the algorithm introduced artificial holes in the pore network skeleton.

The logic of the sandstone modelling system offers, however, a solution to the 3-D skeleton or thinning problem. The inverse function of thinning is dilation. An ultimate thinning of the pore network is therefore exactly the same as an ultimate dilation of the complementary grain network. This is carried out by first numbering each grain and then subjecting the numbered sandbed array to an ultimate dilation of the grains.

In the original mineral matrix space, the border surface between two ultimately dilated grains is a grain-to-grain connection surface. Inside the original pore space, however, this surface contains the pore network skeleton. The skeleton of the pore network is thus found where two or more such dilated grain border surfaces meet. This line is defined by the points in space which have neighbour voxels from 3 or more different ultimately dilated grains. The points on this spatial line which have neighbour voxels from $\mathbf{4}$ or more different ultimately dilated grains are the nodes in the pore network.

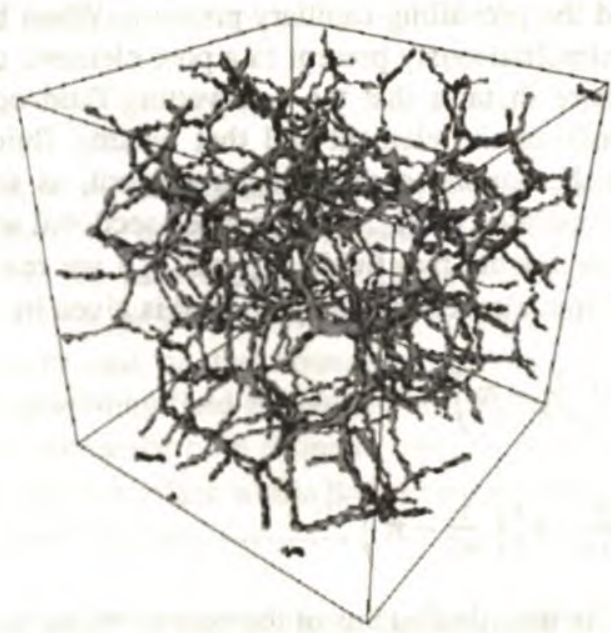

Fig. 9. The network skeleton of the pore space shown in Fig. 8(b).

This ultimate dilation technique provides an excellent estimate of the pore network skeleton (Fig. 9). All the nodes (pore bodies) in the skeleton are mapped and defined by their spatial co-ordinates. The architectural analysis of the pore network is completed with mapping of all the nodes which are connected to each node (i.e. co-ordination number).

Pore geometry. The entire void volume of the sandstone model is allocated to the pore bodies. The volume of each pore body is calculated using an average radius from its geometrical centre point. The pore body wall is searched with a rotating radius vector in increments of 10 degrees 36 times in a plane which is rotated 18 times in increments of 10 degrees (Fig. 10). Both the radii and the co-ordinates of the points where the searching vector hits the pore wall are recorded. Abnormal long radii are assumed to be measured into pore throats and are eliminated. For every pore body, the volume and the inscribed sphere radius $r_{p}$ are recorded.

The pore throats are mapped between the nodes. For each pore throat skeleton voxel, the pore throat wall is mapped in a plane normal to the local direction of the pore throat. The pore throat radius is measured in a manner similar to that of the pore body radius. A rotating vector measures the radius from the skeleton voxel to the pore throat wall in the plane for each 10 degree 36 times (Fig. 10). For every pore throat, the inscribed radius $r_{t}$ of the minimum throat constriction is recorded and used to define drainage threshold capillary pressures.

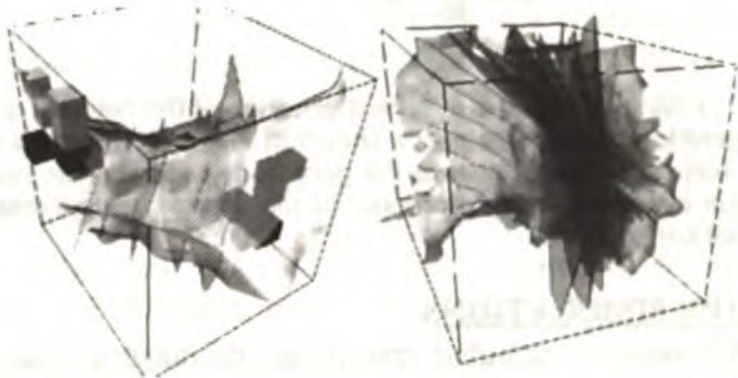

Fig. 10. Geometrical analysis of pore throats (left) and pore bodies (right). In the pore throat image, the light grey transparent surface shows the pore throat wall, the cubes represent the skeleton, and the 6 medium grey transparent planes show how the pore throat mapping is performed. In the pore body image, the light grey transparent surface shows the pore body wall and the 18 rotated medium grey transparent planes illustrates how the pore body geometry is mapped.

A key characteristic of the pore structure of real porous media is the angular corners of pores ${ }^{40}$. The angular corners retain wetting fluid and allow two or more fluids to be flowing simultaneously through the same pore (see below). Pores which are angular in cross-section are thus a much more realistic description of the geometry of pore bodies and throats than the commonly applied cylindrical pore model. In the present work, the crosssectional shape of every pore body and throat is described in terms of a dimensionless shape factor, $G$, which is defined $\mathrm{as}^{40}$

$$
G=A / P
$$

where $A$ is the average cross-sectional area of the pore body or throat and $P$ is the corresponding perimeter length. The value of the shape factor ranges from zero for a slit shaped pore to 0.0481 for a pore having the shape of an equilateral triangle. For a square pore, the shape factor acquires the value 0.0625 .

The analysing technique described above provides a complete and quantitative characterisation of the network architecture of the pore space and the geometry of the pore bodies and throats. Fig. 11 shows a ball-andstick illustration of a sub-sample of the network representation of the pore space shown in Fig. 8(b). 


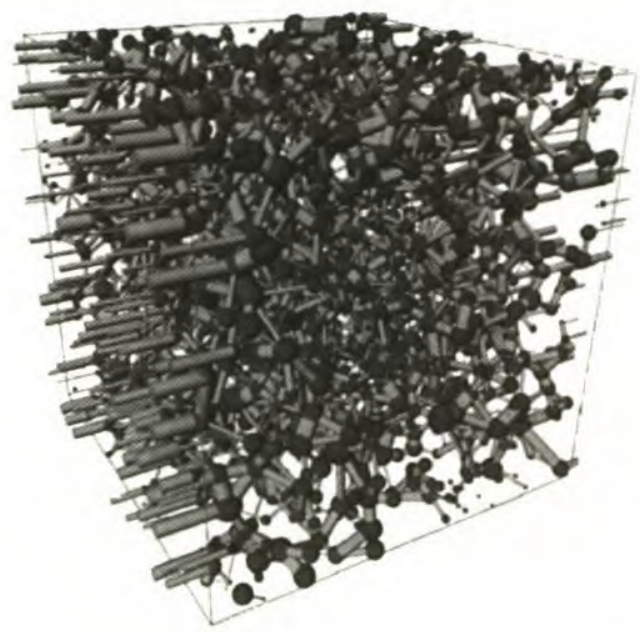

Fig. 11. Simplified ball-and-stick illustration of the network representation of the pore space shown in Fig. 8(b). The radil of the balls are proportional to the pore body radil and the radii of the cylinders are proportional to the size of the minimum throat constrictions.

\section{ELOW SIMULATIONS}

We want to simulate two-phase drainage displacements in the network representations of our sandstone models. Non-wetting fluid is injected into a network which is originally full of wetting fluid. For the present discussion, the wetting fluid completely wets the pore surface. Mixed wet systems are beyond the scope of the present discussion. Two-phase flow in the network is modelled using the following assumptions:

1. The flow is everywhere laminar (Poiseuille flow) and all the fluids are Newtonian, incompressible, and immiscible. The injected fluid is the non-wetting fluid.

2. All the fluid volumes are allocated to the pore bodies whilst all the pressure drops are allocated to the throats connecting the pore bodies.

3. Pore bodies and pore throats may be occupied by one or both fluids simultaneously. In the case of dual occupancy, non-wetting fluid is the bulk fluid whilst wetting fluid is retained as wetting films in angular corners of pore bodies and throats.

4. Pore bodies or nodes are divided into two groups full nodes and interface nodes. Full nodes, i.e. nodes not being invaded, are occupied by a single bulk fluid whilst interface nodes, i.e. nodes undergoing invasion, are occupied by two bulk fluids. The fluid contents of an interface node change with time.

5. Fluid pressures are only defined in pore bodies. Pore bodies are considered to be sufficiently large for the capillary pressure across an invading interface in the pore body to be negligible.

Invasion of pore throats and bodies. When the invading non-wetting fluid is located in a pore body $I$ which is connected to a pore throat $I J$ filled with wetting fluid, capillary forces prexent the non-wetting fluid from spontaneously entering the throat. The non-wetting fluid can only enter the throat when the pressure in the nonwetting fluid $\left(P_{m}\right)$ exceeds the pressure in the wetting fluid $\left(P_{n}\right)$ by a value equal to the threshold capillary pressure $P_{c}$, i.e.

$$
P_{n w, I}-P_{w, J}>P_{c, L J}=\frac{\sigma\left(1+2 \sqrt{\pi G_{L}}\right)}{r_{L}}
$$

where $\sigma$ is the interfacial tension. When the above condition is satisfied, the throat is said to be open to the invading fluid and the invading fluid immediately enters the throat. Otherwise, the throat is closed and the interface remains stationary at the entrance to the throat.

When a throat $I J$ opens, the invading interface immediately enters the adjoining node $J$ which then becomes an interface node. If the interface node is occupied by nonwetting fluid, coalescence occurs and the displacement is considered to be complete. If it is filled with wetting fluid, non-wetting fluid displaces wetting fluid from the node.

Fluid conductivity. The hydraulic conductivity of a fluid in a pore element (pore body or throat) is determined by the geometry of the cross-sectional area open to flow and the prevailing capillary pressure. When both fluids are simultaneously present in a pore element, capillary pressure dictates that the non-wetting fluid occupies the bulk of the element and that wetting fluid is retained in the angular corners of the element, as schematically illustrated in Fig. 12. The cross-sectional areas open to flow for the two fluids $\left(A_{w}\right.$ and $\left.A_{m o}\right)$ are readily calculated from elementary geometry and is given by

$$
\begin{aligned}
& A_{w}=r_{w}^{2}\left(\frac{1}{4 G}-\pi\right) \ldots \ldots \ldots . . . . . \\
& A_{n w}=\frac{r^{2}}{4 G}-r_{w}^{2}\left(\frac{1}{4 G}-\pi\right) .
\end{aligned}
$$

where $r$ is the inscribed radius of the pore element (i.e. $r_{p}$ or $\left.r_{t}\right)$ and $r_{w}$ is the radius of curvature of the film interface which is given by the Young-Laplace equation

$$
r_{w}=\sigma /\left(P_{n w}-P_{w}\right)
$$

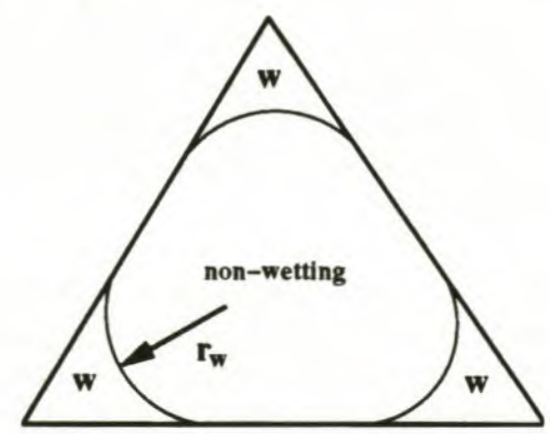

Fig. 12. Distribution of wetting and non-wetting filuids in a triangular pore. Non-wetting fluid occupies the bulk of the pore whilst wetting flidi is retained in the angular corners. 
In the present treatment, we make the simplification that the conductivity of the fluid $i$ which occupies the bulk of a pore element may be characterised by the concept of a mean hydraulic radius, $R_{i}$, defined as

$$
R_{i}=0.5\left(r+r_{v, i}\right)
$$

where $r_{v, i}$ is an equivalent volume radius which is defined as the radius of the cylinder whose volume is equal to the volume of fluid $i$ in the element, i.e.

$$
r_{v, i}=\sqrt{\frac{A_{i}}{\pi}}
$$

If the bulk of the pore element is occupied by nonwetting fluid, $A_{i}$ is given by Equation (6) whilst if it is completely filled with wetting fluid, $A_{i}=r^{2} / 4 G$. The conductivity of fluid $i, g_{i}$, is given by Poiseuille's law

$$
g_{i}=\frac{R_{i}^{2} A_{i}}{8 \mu_{i} l}
$$

where $\mu_{i}$ is the fluid viscosity and $l$ is the length of the pore element.

If wetting fluid is present in the comers of a pore element which is predominantly occupied by non-wetting fluid, we use:

$$
g_{w}=\frac{r_{v, w}^{2} A_{w}}{8 \beta \mu_{w} l}
$$

where $\beta$ is a dimensionless flow resistance factor which accounts for the reduced conductivity of wetting fluid close to the pore wall. Finite element solutions ${ }^{41}$ of the corner flow problem show that $\beta$ depends on the corner geometry and on the boundary condition at the nonwetting/wetting fluid interface. For instance, for an equilateral triangular pore element, $\beta=5.3$ if there is no flow at the fluid interface whilst $\beta=2.5$ for a no stress interface boundary condition.

Phase pressures. Assuming Poiseuille flow, the volumetric flow rate of fluid $i$ between two connecting pore bodies $I$ and $J, Q_{i, J}$ is given by

$$
Q_{i, I J}=g_{i, J}\left(P_{i, I}-P_{i, J}\right)
$$

where $g_{i, I}$ is the hydraulic conductivity of fluid $i$ between the centres of pore bodies $I$ and $J$. For simplicity, we assume that $g_{i \prime \prime}$ is the harmonic mean of the conductances of the throat connecting the pore bodies and the two pore bodies themselves, i.e.

$$
\frac{1}{g_{i, I J}}=\frac{1}{g_{i,}}+\frac{1}{2}\left(\frac{1}{g_{i, 1}}+\frac{1}{g_{i, J}}\right) .
$$

Since the fluids are incompressible, continuity requires that at each node

$$
\Sigma_{J} Q_{i, J}=0
$$

where $J$ runs over all the pore throats connected to node I. Equations (12) and (14), together with the appropriate initial and boundary conditions, give rise to a set of linear algebraic equations for the nodal or pore body centre pressures which in matrix form may be written as

$$
\mathbf{G P}=\mathbf{b}
$$

where $\mathbf{G}$ is a sparse matrix whose elements contain the $g$ 's, $\mathbf{P}$ is the (unknown) nodal pressures, and $b$ is a source vector which is essentially zero except for inlet, outlet, and interface nodes. The nodal pressures are determined by an iterative conjugate gradient method.

The advance of the displacement is treated using the usual quasi-static approximation. During a time step $\Delta t$, the fluids flow through the network at a constant rate in response to the computed pressure fields in each of the two fluids. The time step is selected such that only one interface node is completely filled in every time step. Since a number of interface nodes may be undergoing invasion simultaneously, we identify all the nodes which are filling and compute the time required to fill each node. The minimum invasion time, $\Delta t$, is determined and the non-wetting fluid saturation, $S_{m w}(t)$, in every interface node is updated, i.e.

$$
S_{m w, I}(t+\Delta t)=S_{n w, I}(t)+\frac{\Delta l}{V_{I}} \Sigma_{J} Q_{I J}
$$

where $V_{l}$ is the volume of node $I$ and the sum only includes non-wetting phase filled pore throats which are connected to interface node $I$. In some interface nodes, $S_{n w}$ may decrease. If $S_{n w}$ reaches zero, non-wetting fluid spontaneously retracts out of the connecting pore throats and the node is removed from the list of interface nodes.

Initial and boundary conditions. Initially, the network contains only wetting fluid. Non-wetting fluid is injected at a constant rate through a non-wetting fluid reservoir which is attached via pore throats to every pore body along the inlet side of the network. Fluids escape through the outlet face on the opposite side of the network where the wetting phase pressure is arbitrarily' set to zero. Impermeable boundary conditions are imposed along the sides parallel to the main direction of flow.

Since the wetting fluid is continuous throughout the entire network, Equation (15) must be applied at all pore bodies other than those at the outlet where the pressure is arbitrarily set to zero. The non-wetting fluid is also continuous. However, it only occupies part of the network at any instant in time. Equation (15) is thus only applied at pore bodies which are occupied by the non-wetting fluid.

The solution for the two fluid pressures is iterative because the individual fluid pressures are coupled through the pressures in interface nodes where the bulk fluid pressures are equal, and because it is necessary to specify a priori which pore throats are open and which are closed to the invading non-wetting fluid. The procedure for each time step, $\Delta t$, is as follows: 
1. The pressure for each fluid in every node containing the fluid is computed using Equation (15).

2 . The computed pressures are used to calculate capillary pressures for each closed throat. If the capillary pressure exceeds the threshold pressure, the throat is opened and the pressure solution is re-calculated to check that the throat remains open. This procedure is iterated until the computed capillary pressures are everywhere consistent with the threshold capillary pressures.

3. The flow rates at every interface node is computed using Equation (12). The flow rates are used to calculate the time step $\Delta t$ such that only one interface node is completely filled during the time step.

4. The saturation in all interface nodes is updated using Equation (16).

5. Repeat the above procedure for the next time step.

Macroscopic properties. The calculations of macroscopic transport properties are performed on a central region of the pore network in order to minimise boundary effects. When the network is fully saturated with wetting fluid, we specify a constant pressure drop across the network and calculate the flow rate $Q$ and the pressure drop $\Delta P$ across the region of interest. The absolute permeability $K$ is determined from Darcy's law, i.e.

$$
K=\frac{Q \mu L}{A \Delta P}
$$

where $A$ is the cross-sectional area of the domain and $L$ is the length.

Relative permeabilities, $k_{r}$ are calculated in a similar manner. At various stages of the displacement we compute the average pressure drop in the non-wetting and wetting fluids across the domain. The saturation and the total flow rates of non-wetting and wetting fluids across the domain are also calculated. The relative permeability of fluid $i$ is computed using Darcy's law

$$
k_{r, i}(S)=\frac{Q_{i} \mu_{i} L}{K A \Delta P_{i}}
$$

The effective capillary pressure, $P_{c}(S)$, in the domain is defined as the difference in the mean pressures of the non-wetting and wetting fluids. This is computed at various stages of the simulation.

\section{RESULTS}

Bourbie and Zinszner ${ }^{\wedge}$ performed extensive laboratory measurements of the hydraulic and acoustic properties of Fontainebleau sandstone. Thin section analysis reveals that the grain-size distribution of this essentially pure quartz sandstone is quite narrow with an average grain size of around $200 \mu \mathrm{m}$ (within a $10-20 \%$ boundary) ${ }^{29,42}$. The porosity and permeability, however, vary widely. Porosity ranges from $2 \%$ to $30 \%$ without noticeable changes in grain size whilst the permeability spans nearly five orders of magnitude ${ }^{42}$. The variation in these properties is due almost entirely to the degree of quartz cementation $^{29}$. Quartz cementation is reasonably approximated by the uniform grain overgrowth model described earlier. It thus provides a convenient test of our sandstone models and their network representations.

Fig. 13(a) shows a microtomographic image of a small cube of Fontainebleau sandstone whilst Fig. 13(b) shows an analog Fontainebleau sandstone model generated by the procedure described above. The corresponding pore space images are shown in Figsi 14 (a) and (b), respectively. The linear scale of the images is 150 voxels (or $1.125 \mathrm{~mm}$ ) on a side. Despite the necessarily approximate treatment of the complex geological processes which govern the formation of sandstones, the simulated sandstone model is notably similar to that of the real Fontainebleau sandstone. The volume fraction of the pore space is $13.5 \%$ for both models. The main discrepancies between the model sandstone and the real one arise because the rock grains are not spherical.
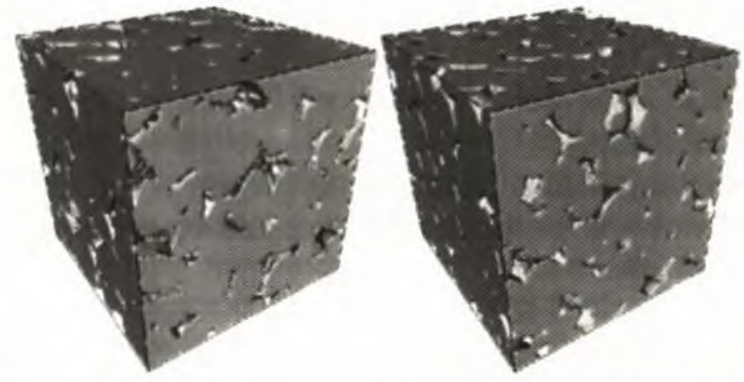

Fig. 13. Comparison between (a) $\mu-\mathrm{CT}$ image of a Fontainebleau sandstone (left) and (b) simulated Fontainebleau sandstone model (right).

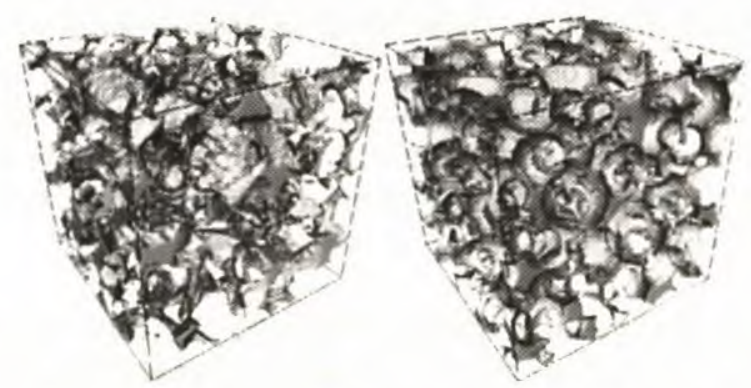

Fig. 14. (a) $\mu-C T$ image of the pore space of the Fontainebleau sandstone shown in Fig. 13 (a), and (b) the pore space of the simulated sandstone model shown in Fig. 13 (b).

Using the simulated Fontainebleau sandstone model shown in Fig. 13(b) as basis, we generated a total of 9 different models by varying the degree of quartz cementation. The computed permeabilities of the network representations of these models are compared with a series of 240 measurements of Fontainebleau sandstone performed by Bourbie and Zinszner ${ }^{\wedge 2}$ (Fig. 15). Although the measured permeabilities span nearly five orders of magnitude, the simulated permeability versus porosity trend is in good agreement with the measured one. We emphasise that this agreement is obtained without using any adjustable parameters. The only inputs have been average grain size (prior to compaction and cementation), 
sorting, and knowledge that porosity reduction is caused by quartz cementation alone.

The above results demonstrate that the sandstone models and their network representations, including the assignment of conductivities, adequately predict the average behaviour in single-phase flow.

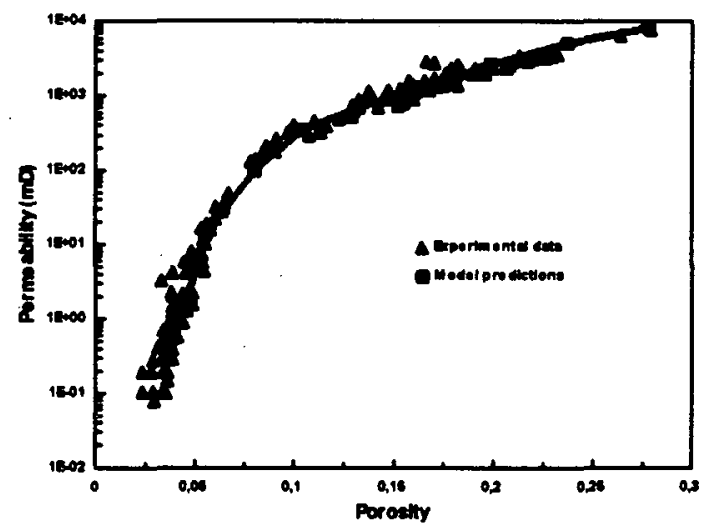

Fig. 15. Comparison between predicted and measured absoIute permeability for Fontainebleau sandstone.

Relative Permeability. Șteady state drainage relative permeability measurements were performed on two "twin" samples of a strongly water wet Bentheimer sandstone. In order to minimise experimental uncertainties, two repeat measurements were performed on each sample (i.e. a total of 4 measurements) and a polynomial regression curve was fitted to the data.

Thin section images of the two samples were analysed and used to build four realisations of an analog sandstone model. A capillary dominated (i.e. $N_{c a}=10^{-6}$ ) drainage displacement was simulated on the network representation of each of the four realisations and average relative permeabilities were computed at various stages of the simulations. The value of the comer flow resistance factor $\beta$ in these simulations was 2.5 which is appropriate for pure fluids.

Fig. 16 compares predicted and measured relative permeabilities. The predicted relative permeabilities are in good agreement with those determined experimentally, both for the wetting and non-wetting fluids. Fig. 17 shows that the experimentally measured (porous plate method) and computed capillary pressures also are in good agreement. Again, we emphasise that this agreement is obtained without the use of any adjustable parameters. The only inputs are readily available thin section data and knowledge that capillary forces dominate the displacement on the pore-scale.

The above results are encouraging and suggest that the present methodology for generating sandstone models which are analogs of real sandstones may be used to extend network modelling techniques to real porous media. This offers the possibility of predicting macroscopic (cm-scale) transport properties from the underlying porescale physics and to elucidate how these properties de- pend on geological factors such as porosity, compaction, and different diagenetic processes.

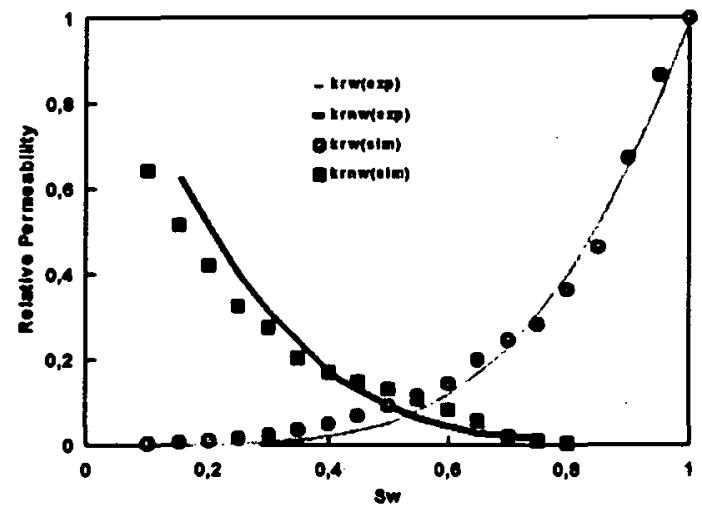

Fig. 16. Comparison between simulated and experimentally measured drainage relative permeabilities for a strongly water wet Bentheimer sandstone.

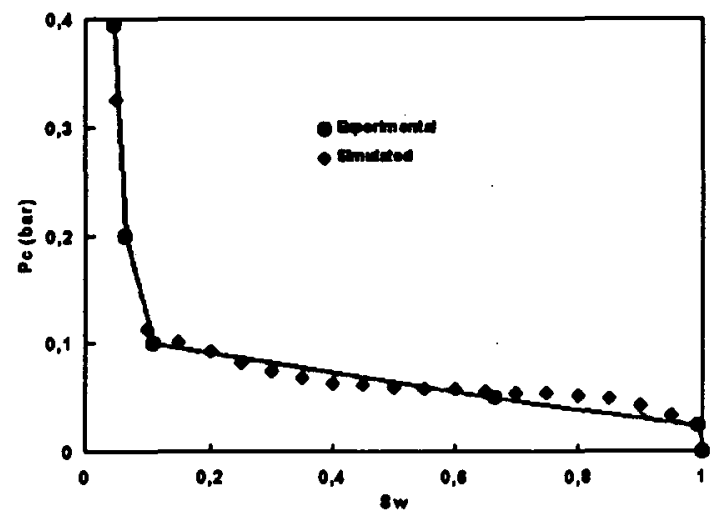

Fig. 17. Comparison between simulated and experimentally measured drainage capillary pressure for a strongly water wet Bentheimer sandstone.

\section{CONCLUSIONS}

1. Process/stochastic modelling of the sandstone forming processes sedimentation, compaction, and diagenesis yields physically representative sandstone models -which are analogs of actual sandstones.

2. 3-D image analysis algorithms may. be used to extract the network representation of the pore space for a sandstone model from its complementary grain matrix network.

3. Predicted absolute permeabilities matches measurements on Fontainebleau sandstone samples having permeabilities spanning nearly five orders of magnitude.

4. Using a network model which accounts for both viscous and capillary forces, we accurately predict drainage relative permeability and capillary pressure for a strongly water wet Bentheimer sandstone.

\section{Acknowledgements}

The authors are indebted to Svein H. Midtlyng who performed the experimental work. We also graciously thank David Stern, Exxon Production Research Company, for 
providing the microtomography data. The authors would like to acknowledge Den norske stats oljeselskap a.s. (Statoil) for granting permission to publish this paper.

\section{References}

1. Fatt, I.: "The Network Model of Porous Media I. Capillary Pressure Characteristics," Trans AIME, 207 (1956), 144-159.

2. Fatt, I.: "The Network Model of Porous Media II. Dynamic Properties of a Single Size Tube Network," Trans AIME, 207 (1956), 160-163.

3. Fatt, I.: "The Network Model of Porous Media III. Dynamic Properties of Networks with Tube Radius Distribution," Trans AIME, 207 (1956), 164-181.

4. Larson, R.G., L.E. Scriven, and H.T. Davis:"Percolation theory of two-phase flow in porous media", Chem Eng. Sci., 36 (1981), 57-73.

5. Koplik, J.: "Creeping flow in two-dimensional networks", J. of Fluid Mechanics., 119 (1982), 219-247.

6. Wilkinson, D. and J.F. Willemsen: "Invasion percolation: a new form of percolation theory", J. Phys. A., 16 (1983), 3365-3376.

7. Koplik, J. and T.J. Lasseter: "Two-Phase Flow in Random Network Models of Porous Media", SPEJ, 22 (1985), 89-100.

8. Chan, D.Y.C., B.D. Hughes, L. Paterson, and C. Sirakoff: "Simulating flow in porous media", Physical Review A, 38 (1988), 4106-4120.

9. Lenormand, R., E. Touboul, and C. Zarcone: "Numerical models and experiments on immiscible displacements in porous media", J. Fluid Mechanics, 189 (1988), 165-187.

10. Payatakes, A.C.: "Three-Dimensional Network Model for Consolidated Porous Media. Basic Studies", Chem. Eng. Communications, 81 (1989), 55

11. Blunt, M.J. and P.R. King: "Macroscopic Parameters from Simulation of Pore Scale Flow," Phys. Rev. A 42, (1990), 4780-4788.

12. Jerauld, G.R. and S.J. Salter: "The effect of pore-structure on hysteresis in relative permeability and capillary pressure: pore-level modelling", Transport in Porous Media, 5 (1990) 103-151.

13. Blunt, M.J. and P.R. King: "Relative permeabilities from two- and three-dimensional pore-scale network modelling", Transport in Porous Media, 6 (1991), 407-433.

14. Blunt, M.J., M. King, and H. Scher: "Simulation and theory of two-phase flow in porous media", Phys. Rev. A, 46 (1992), 7680-7699.

15. Bryant, S. and M.J. Blunt: "Prediction of Relative Permeability in Simple Porous Media", Phys. Rev. A, 46 (1992), .2004-2011.

16. Bryant, S., P.R. King, and D.W. Mellor: "Network model evaluation of permeability and spatial correlation in a real random sphere packing", Transpon in Porous Media. 11 (1993), 53-70.

17. Billiotte, J., H. De Moegen, and P.E. Øren: "Experimental micromodelling and numerical simulation of gas-water injection-withdrawal cycles as applied to underground gas storage", SPE. Adv. Tech. Ser., 1 (1993), 133-139.

18. McDougall, S.R. and K.S. Sorbie: "The combined effect of capillary and viscous forces on waterflood displacement efficiency in finely laminated porous media", SPE 26659. proceedings of the SPE Annual Techincal Conference and Exhibition, Houston, (1993).

19. Soll. W.E. and M.A. Celia: "A Modified Percolation Approach to Simulating Three-Fluid Capillary PressureSaturation Relationships", Advances in Water Resources, 16 (1993), 107-126.

20. Øren, P.E., J. Billiotte, and W.V. Pinczewski: "Pore-scale network modelling of waterflood residual oil recovery by immiscible gas flooding". SPE-27814, proceedings of the SPE/DOE 9th Symposium on IOR, Tulsa, (1994).

21. McDougall, S.R. and K.S. Sorbie: "The Impact of Wettability on Waterflooding: Pore-Scale Simulation", SPE Resevoir Engineering, 10 (1995), 208-213.

22. Blunt, M.J. and H. Scher: "Pore Level Modelling of Wetting," Physical Review E, 52, (1995), 6387-6403.

23. Fenwick, D.H. and M.J. Blunt:, "Pore level modelling of three phase flow in porous media", proceedings of the 8th European IOR Symposium, Vienna, Austria, (1995).

24. Øren, P.E. and W.V. Pinczewski: "Fluid distribution and pore-scale displacement mechanisms in drainage dominated three-phase flow", Transport in Porous Media, 20 (1995),105-133.

25. Pereira, G.G., W.V. Pinczewski, D.Y.C. Chan, L. Paterson, and P.E. Øren: "Pore-scale network model for drainage dominated three-phase flow in porous media", to appear in Transport in Porous Media (1996).

26. Coles, M.E., P. Spanne, E.L. Muegge, and K.W. Jones: "Computed Microtomography of Resevoir Core Samples," proceedings of the International SCA Meeting, Stavanger. Norway, (1994).

27. Holt, R. M., E. Fjær, O. Torsæter, and S. Bakke.: "Petrophysical Laboratory Measurements for Basin and Reservoir Evaluation", to appear in Marine and Petroleum Geology, (1996).

28. Lin, C. and M.H. Cohen: "Quantitative Methods of Microgeometric Modelling," J. Appl. Phys. 53 (1982), 4152.

29. Bryant, S., C. Cade, D. and Mello: "Permeability Prediction from Geologic Models," AAPG Bulletin, 77 (1993) 1338-1350.

30. Finney, J.: "Random Packings and the Structure of Simple Liquids. I. The Geometry of Random Close Packings", Proc. Roy. Soc., 319A. (1970), 479.

31. Adler, P. M.: Porous Media Geometry and Transports. Butterworth-Heinemann Series in Chemical Engineering (1992).

32. Ehrenberg, S.N. and T. Boassen: "Factors Controlling Permeability Variation in Sandstones of the Garn Formation in Trestakk Field, Norwegian Continental Shelf", J. of Sedimentary Petrology. 63 (1993), 929-944.

33. Sundborg, $\AA .:$ "The River Klaraalven, a Study in Fluvial Processes," Geografiska Annaler, Serie A, 38 (1956), 125.

34. Bakke, S. and P.E. Øren: "3-D Pore-Scale Modelling of Heterogeneous Sandstone Reservoir Rocks and Quantitative Analysis of the Architecture, Geometry and Spatial Continuity of the Pore Network", SPE 35479, proceedings of the European 3-D Reservoir Modelling Conference. Stavanger, Norway, (1996).

35. Bjorkum,P. A.: "How Important is Pressure in Causing Dissolution of Quartz in Sandstones?", J. Sed. Res., In press.

36. Ferreol, B. and D.H. Rothman: "Lattice-Boltzmann Simulations of Flow through Fontainbleau Sandstone", Transport in Porous Media, 20 (1995), 3-20. 
37. Serra, J.: "Image analysis and mathematical morphology." Academic Press, New York (1982).

38. Hilditch, C. J.: "Linear skeleton from square cupboards," in Machine Inselligence 6, Edinburgh Univ. Press, (1969) 403.

39. Thovert, J. F., J. Salles, and P.M. Adler: "Computerized Charcterization of the Geometry of Real Porous Media: Their Discretization, Analysis and Interpretation", J.of Microscopy, 170, (1993), 65.

40. Mason, G. and N.R. Morrow: "Capillary behaviour of a perfectly wetting liquid in irregular triangular tubes," $J$. of Colloid and Interface Science, 141 (1991), 262-274.

41. Ranshoff, T.C. and C.J. Radke: "Laminar Flow of Wetting Liquid along the Corners of a Predominantly Gas-Occupied Noncircular Pore", J. of Colloid and Interface Science, 121 (1988), 392-401.

42. Bourbie, T. and B. Zinszner: "Hydraulic and Acoustic Properties as a Function of Porosity in Fontainebleau Sandstone", J. of Geophysical Research, 90 (1985), 11524-11532. 
